\section{The Professional Counselor: Author and Article Characteristics From 2011 to 2019}

\author{
Dorrie Williams, Marcella Melanson, Bradley T. Erford
}

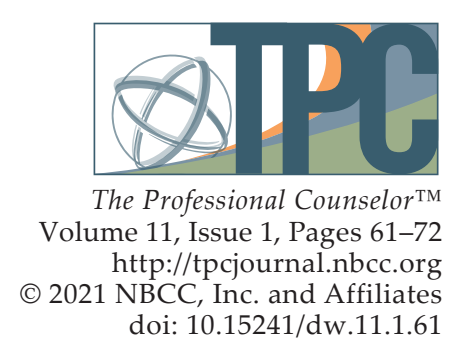

This meta-study evaluates publication patterns and trends occurring in the first 9 years of The Professional Counselor (TPC). Both author (e.g., gender, domicile, employment setting, top individual and university contributors) and article characteristics (e.g., topic, research design, participant type, sample size, statistics) are identified, with a particular focus on research articles. Almost $64 \%$ of lead authors and all authors were women, $92.1 \%$ of lead authors were affiliated with universities, and $3.4 \%$ of lead authors were internationally domiciled. From 2011-2019, the University of Central Florida featured the greatest number of lead authors, and the top author overall was Dr. Kathleen Brown-Rice. About 58\% of published works were research articles, and of those, $69 \%$ used quantitative design methodology. Nearly all coded research variables were stable over time, except for participant types, as the proportion of adult participant samples increased while undergraduate participant samples decreased over time.

Keywords: meta-study, author characteristics, article characteristics, publication patterns, quantitative design

The Professional Counselor (TPC) is an electronic journal published by the National Board for Certified Counselors (NBCC; The Professional Counselor, n.d.). Beginning publication in 2011, this peer-reviewed and open-access journal publishes research and practice-based articles related to school counseling, marriage and family counseling, counseling supervision, theory development, counseling ethics, mental and behavioral health counseling, and multicultural and international counseling. The primary goal of this quarterly journal is to provide relevant and interesting resources and information for individuals all across the counseling profession, including counselors, counselor educators, mental health practitioners, graduate students, and supervisors. Thus, TPC is meant to address the professional development and informational needs of all counselors, regardless of specialty, work setting, or discipline. Each issue of TPC is released alongside a digital reader's digest, a collection of summaries of the articles that are included within the issue.

Myriad characteristics within the counseling profession have evolved over the last decade, and along with this evolution, counseling research also has changed. Just as it is important to periodically review changes to the counseling profession, it also is vital to review journal characteristics to observe and analyze how journals evolve in relation to societal and cultural changes (Erford et al., 2010). Such an analysis informs the editorial board, authors, and readership about who and what is published in a journal, characteristics of methodological considerations, and the trajectories of those characteristics. As TPC began publication in 2011, this is the first multi-year review of the journal's characteristics, providing an opportunity to examine trends within this journal that have yet to be studied. Historical reviews such as this allow for longitudinal monitoring of the advancement of, and challenges occurring within, the counseling profession.

Erford et al. (2010) identified several viable processes for analyzing and recording journal publication patterns. These methods include content analysis of published special issues and sections, qualitative synthesis, and quantitative meta-studies. From 2011-2019, five special issues were published in

Dorrie Williams is a master's candidate at Vanderbilt University. Marcella Melanson is a master's candidate at Vanderbilt University. Bradley T. Erford, PhD, NCC, LPC, LCPC, is a professor in the Peabody College at Vanderbilt University. Correspondence may be addressed to Bradley T. Erford, PMB 90, Vanderbilt University, Nashville, TN 37202-5721, Bradley.t.erford@vanderbilt.edu. 
TPC - Counseling the Military and Their Families (Volume 4, Issue 2); Counseling and the DSM-5 (4.3); School Counseling (4.5); Counseling Children With Special Needs and Circumstances (5.2); and School Counselors and a Multi-Tiered System of Supports: Cultivating Systemic Change and Equitable Outcomes (6.3) - along with one special section (Counselor Education and Supervision [5.1]). To date, no qualitative syntheses of TPC content have been published in TPC. Qualitative syntheses are usually conducted by a scholar with expertise in qualitative analysis and provide an in-depth scholarly treatise of the content published by a journal.

This current article is the first meta-study of TPC content. A meta-study involves conducting a quantitative review and trend analysis of patterns found within the published literature. Quantitative meta-studies typically include an analysis of the author and article characteristics, as well as the use of descriptive and univariate statistical procedures in order to identify trends over time within the characteristics analyzed. These studies can be as narrowly focused as a single journal or topic or can span across multiple journals focusing on specific concepts. Quantitative meta-studies have the advantage of allowing objective, numerical, statistical analysis of changing trends across a large variety of variables using hypothesis testing to determine statistical significance and effect sizes. This quantitative meta-study focused on the scholarly content of TPC from the first nine volumes (20112019) and attempted to answer two basic questions: (a) What is published within The Professional Counselor (article characteristics), particularly with regard to research? and (b) Who publishes in The Professional Counselor (author characteristics)? These questions are also analyzed for trends to determine changes occurring in journal characteristics over time.

\section{Method}

Mirroring the methods of Saks et al. (2020), all articles published from 2011 through 2019 in TPC were examined, analyzed, and coded to describe article and author characteristics in order to answer the primary research questions of who and what is published in TPC and how those characteristics have changed over time. This systematic approach also allowed comparisons of these variable displays across journals. Scholarly contributions were accepted into the analysis while less scholarly works were rejected and not included in the analysis (e.g., editorials, introductions to special issues, biographies, profiles). Identified author characteristics included the number of authors, name, gender, and employment setting of all authors, and university and domicile (national or international) of the lead author. Identified article characteristics included type of article (i.e., expository/other and research), topical content (e.g., professional issues, symptoms/disorders, technique/theory, multicultural issues), and focus (i.e., research or not research). Additional characteristics were identified specifically in research articles. These research characteristics included: intervention or nonintervention; research paradigm (i.e., quantitative or qualitative); type of research design (i.e., qualitative, true or quasiexperimental, test development, descriptive/survey, comparative, correlational, meta-analysis/ other); use of random or nonrandom sampling/assignment procedures; types of participants (i.e., adults, counselors/providers, youth, undergraduate students, graduate students or counselor trainees, nonhuman); sample size; sample size category (i.e., small, medium, large, or very large); sophistication of statistical applications (basic, intermediate, or advanced); primary statistical analyses used (i.e., descriptive, correlation, regression analysis, $t$-test/ANOVA/ANCOVA, nonparametric, MANOVA/MANCOVA, factor analysis); and inclusion of sample effect size estimate, reliability, and validity as indicators of quantitative reporting standards. Qualitative designs (e.g., grounded theory, phenomenological, case study) were further disaggregated and analyzed. 
Our first two authors, Dorrie Williams and Marcella Melanson, independently coded each article for these characteristics into separate Microsoft Excel spreadsheets, sequenced by article release date. The data were then assessed for discrepancies and resolved by consensus. Analysis included descriptive statistics and one-way ANOVA using weighted proportions to analyze trends over time. Post hoc tests of significant results were conducted using Scheffe tests and significance for Type I error was set at $\alpha<.05$. The interpretive guidelines of $.01=$ small effect, $.09=$ medium effect, and $.25=$ large effect were used to report ANOVA effect sizes $\left(\eta^{2}\right)$. These effect size estimates help demonstrate the power or relevance of the observed effects; larger effect sizes indicate more important or relevant changes being observed.

\section{Results}

From 2011-2019, 272 articles were published in TPC. Of these, 265 articles were accepted into this analysis, with only seven articles rejected because they were less scholarly submissions (e.g., editorials, introductions to special issues, biographies, profiles). Results were analyzed for trends over time using ANOVA with weighted proportions after being aggregated into two time windows (2011-2014 and 2015-2019). The following results were segmented by author and article characteristics.

\section{Author Characteristics}

Author demographic variables, such as the gender of the lead author and all authors and employment setting and domicile of lead author, were used to assess TPC author characteristics. We also identified the most prolific author contributors and sponsoring institutions and monitored collaboration trends by computing the average number of authors per article. A consistent, solid majority of lead and all authors from 2011-2019 self-identified as women. Women lead authors formed a proportion of $64.2 \%-F(1,263)=0.543, p=.462, \eta^{2}=.002-$ with a similar result for all authors (63.8\% women): $F(1,703)=1.134, p=.287, \eta^{2}=.002$. Reflecting a slight collaboration trend, the average number of authors per article has risen slightly over time. In 2011-2014, TPC reflected an average of 2.43 authors per article, while in 2015-2019, the average increased slightly to 2.83 authors per article.

TPC lead author employment affiliation in university and non-university settings was very stable over time: $F(1,263)=0.953, p=.330, \eta^{2}=.004$. A consistent proportion of $92.1 \%$ of all lead authors were employed or primarily affiliated within a university work setting. During 2011-2019, only $3.4 \%(n=9)$ internationally domiciled lead authors published in TPC: $F(1,263)=2.80, p=.133, \eta^{2}=.009$.

The most frequently contributing individual authors (weighted as the lead author receives 3 points, second author receives 2 points, and subsequent authors receive 1 point each) from 2011-2019 included the following, listed from highest rank to lowest: Kathleen Brown-Rice, with 17 points; Jeffrey M. Warren, with 15 points; Robert C. Reardon, with 12 points; Patrick R. Mullen, with 11 points; Sejal M. Barden, Ashley J. Blount, and Emily Goodman-Scott, with 10 points each; M. Ann Shillingford-Butler, with nine points; Randall M. Moate, with eight points; and Simone Lambert, James P. Sampson, Jr., Richard A. Wantz, and Laura E. Welfare, with seven points each. Using a weighted point system allows more credit to be allotted to primary article contributors over time, whereas a unit weighting system (one point of credit per article authored) benefits contributors who served in secondary or tertiary capacities. From 2011-2019, the following universities supported the highest number of TPC lead authors, listed from highest to lowest: the University of Central Florida, with 12 articles; the University of South Dakota, with 10 articles; Virginia Tech, with six articles; East Carolina University, Florida State University, University of North Carolina - Charlotte, and University of North Carolina - Pembroke, each with five articles; and Wake Forest University and Walden University, with four articles each. 
These universities align well with productive contributing authors in the list above or clusters of contributing authors, representing counseling faculty and counselor education programs with high degrees of dedication to enhancing the counseling literature in TPC.

\section{Article Characteristics}

Table 1 shows that topical content appearing in TPC publications from 2011-2019 was consistent and diverse: $F(1,530)=0.666, p=.415, \eta^{2}=.001$. The topics of counselor education and training, school counseling, and multicultural issues each occurred in more than 10\% of TPC articles, each benefitting as foci in special issues/sections over the years. When inspecting article typology from the perspective of the tables of contents, it is noted that TPC only designates two major categories of articles: research and expository, the latter of which we combined with an "other" category, as specialized, nonresearch articles do appear in TPC from time to time. This TPC table of contents bi-factor typology was consistent from 2011-2019: $F(1,263)=2.033, p=.155, \eta^{2}=.008$. Given this result, it is then not surprising that TPC also displayed a consistent proportion of published research articles (58.1\% from 2011-2019): $F(1,263)=2.357, p=.126, \eta^{2}=.009$. The remainder of this Results section reviews various characteristics of the 154 research articles published in TPC between 2011 and 2019.

\section{Table 1}

Issue Categories in TPC Articles From 2011-2019

\begin{tabular}{lccc}
\hline Content Topic & $2011-2014$ & $2015-2019$ & Total \\
\hline Counselor Education/Training & $35(16.9 \%)$ & $52(16.0 \%)$ & $87(16.4 \%)$ \\
Multicultural Issues & $26(12.6 \%)$ & $29(8.9 \%)$ & $55(10.3 \%)$ \\
School Counseling & $19(9.2 \%)$ & $36(11.1 \%)$ & $55(10.3 \%)$ \\
Health/Wellness & $15(7.2 \%)$ & $30(9.2 \%)$ & $45(8.4 \%)$ \\
Treatment/Intervention & $11(5.3 \%)$ & $32(9.8 \%)$ & $43(8.1 \%)$ \\
Counseling Process & $7(3.4 \%)$ & $33(10.2 \%)$ & $40(7.5 \%)$ \\
Symptoms/Disorders & $21(10.1 \%)$ & $15(4.6 \%)$ & $36(6.8 \%)$ \\
Professional Issues & $16(7.7 \%)$ & $16(4.9 \%)$ & $32(6.0 \%)$ \\
Ethical/Legal Issues & $10(4.8 \%)$ & $19(5.8 \%)$ & $29(5.4 \%)$ \\
Academic/Career & $7(3.4 \%)$ & $18(5.5 \%)$ & $25(4.7 \%)$ \\
Career Development & $12(5.8 \%)$ & $13(4.0 \%)$ & $25(4.7 \%)$ \\
Assessment/Evaluation & $11(5.3 \%)$ & $13(4.0 \%)$ & $24(4.5 \%)$ \\
Supervision & $10(4.8 \%)$ & $9(2.8 \%)$ & $19(3.6 \%)$ \\
Identity Development & $7(3.4 \%)$ & $10(3.1 \%)$ & $17(3.2 \%)$ \\
\hline Totals & 207 & 325 & 532 \\
\hline
\end{tabular}

Note. Many articles were coded to reflect multiple content issues. Thus, totals exceed the number of accepted articles. 
The proportions of quantitative and qualitative research studies appearing in TPC have changed significantly over time $-F(1,176)=9.025, p=.003, \eta^{2}=.049$ - constituting the highest effect size of any analysis in this study, albeit still a small effect. Quantitative designs shifted from a slight minority (46.9\%) of research designs in 2011-2014 to a substantial proportional majority (69.3\%) in 2015-2019, while the qualitative studies displayed converse proportions. We noted that about one-third of the qualitative studies did not specify an approach or methodological tradition. When qualitative approaches/ methodologies were specified: $15.4 \%$ used a generic "content analysis", 30.7\% were phenomenological, $9.6 \%$ used grounded theory, $7.7 \%$ used case study, $5.8 \%$ used consensual qualitative research, $5.8 \%$ used narrative, $3.8 \%$ used constant comparative, and $1.9 \%$ simply specified an ethnographic approach.

Intervention studies maintained a stable presence among TPC research articles at $12.3 \%$ : $F(1,152)=0.020, p=.889, \eta^{2}=.000$. Likewise, the types of research designs appearing in TPC research articles (see Table 2 ) have been relatively stable over time: $F(1,156)=1.232, p=.269$, $\eta^{2}=.008$. Non-experimental designs dominated TPC research articles and were heavily weighted toward descriptive/survey (42.4\%), qualitative (18.4\%), and correlational designs $(18.4 \%)$. The most rigorous experimental designs (true/quasi-experimental designs) comprised only $4.4 \%$ of TPC research studies.

\section{Table 2}

Proportion of Research Designs Used in TPC Research Studies

\begin{tabular}{lccc}
\hline Time & $2011-2014$ & $2015-2019$ & Total \\
\hline Descriptive/Survey & $22(35.5 \%)$ & $45(46.9 \%)$ & $67(42.4 \%)$ \\
Qualitative & $16(25.8 \%)$ & $13(13.5 \%)$ & $29(18.4 \%)$ \\
Correlation & $17(27.4 \%)$ & $12(12.5 \%)$ & $29(18.4 \%)$ \\
SSRD & $1(1.6 \%)$ & $7(7.3 \%)$ & $8(5.1 \%)$ \\
Comparative & $2(3.2 \%)$ & $5(5.2 \%)$ & $7(4.4 \%)$ \\
True/Quasi-Experiment & $3(4.8 \%)$ & $4(4.2 \%)$ & $7(4.4 \%)$ \\
Meta-Analysis/Other & $0(0.0 \%)$ & $6(6.2 \%)$ & $6(3.8 \%)$ \\
Test Development & $1(1.6 \%)$ & $4(4.2 \%)$ & $5(3.2 \%)$ \\
\hline Totals & 62 & 96 & 158 \\
\hline
\end{tabular}

Note. SSRD = Single-subject research design

Proportions of types of participants have shifted significantly across TPC research studies$F(1,224)=5.573, p=.019, \eta^{2}=.024-$ the second highest effect size of this meta-study. From the 2011-2014 to 2015-2019 time windows, adult participant samples increased from $28.0 \%$ to $41.7 \%$, while undergraduate samples dropped from $17.3 \%$ to $6.0 \%$ (see Table 3). TPC research article sample sizes by category were consistent over time: $F(1,153)=0.901, p=.344, \eta^{2}=.006$. Small samples (<30 participants) composed $33.5 \%$ of all studies, medium samples (30-99 participants) $20.0 \%$, large samples (100-499 participants) $36.1 \%$, and very large samples (500+ participants) only $10.3 \%$ of research studies. At the same time, the median sample size increased from 65 participants in 2011- 
2014 to 107 participants in 2015-2019, commensurate with the slight decrease in qualitative studies. Finally, the proportions of use of randomization in assignment procedures was also consistent$F(1,152)=1.172, p=.281, \eta^{2}=.008$ - holding steady at $11.7 \%$ of studies.

\section{Table 3}

Types of Participants Used in TPC Research Articles

\begin{tabular}{lccc}
\hline Time & $2011-2014$ & $2015-2019$ & Total \\
\hline Adults & $21(28.0 \%)$ & $63(41.7 \%)$ & $84(37.2 \%)$ \\
Counselors/Providers & $14(18.7 \%)$ & $39(25.8 \%)$ & $53(23.5 \%)$ \\
Graduate Students/Trainees & $15(20.0 \%)$ & $19(12.6 \%)$ & $34(15.0 \%)$ \\
Undergraduates & $13(17.3 \%)$ & $9(6.0 \%)$ & $22(9.7 \%)$ \\
Youth & $9(12.0 \%)$ & $15(9.9 \%)$ & $24(10.6 \%)$ \\
Nonhumans & $3(4.0 \%)$ & $6(4.0 \%)$ & $9(4.0 \%)$ \\
\hline Totals & 75 & 151 & 226 \\
\hline
\end{tabular}

Categorization of statistical procedures into basic, intermediate, or advanced designations was used as an indicator of statistical sophistication, which remained stable over time: $F(1,152)=0.141, p=.707$, $\eta^{2}=.001$. Articles were specifically categorized as research and non-research articles, and all types of statistical procedures from Table 4 were aggregated into the collapsed categories of basic, intermediate, and advanced statistics. Based on this categorization, over the 9 years, 59.1\% of TPC research studies used basic statistical procedures (e.g., descriptive, correlation, $t$-test, chi-square), 31.8\% used intermediate statistical procedures (e.g., ANOVA, regression analysis, other nonparametric), and only $9.1 \%$ used advanced procedures (e.g., MANOVA, factor analysis). Likewise, when specific statistical procedures used were analyzed, no significant shifts over time occurred (see Table 4): $F(1,290)=0.055, p=.814$, $\eta^{2}=.000$. Descriptive statistics were used in $29.8 \%$, ANOVA/t in $13.4 \%$, and content analysis or thematic coding in $21.2 \%$ of TPC research studies.

\section{Table 4}

Proportion of Various Statistical Procedures Used in TPC Research Studies

\begin{tabular}{lccc}
\hline Time & $2011-2014$ & $2015-2019$ & Total \\
\hline Descriptive & $29(27.4 \%)$ & $58(31.2 \%)$ & $87(29.8 \%)$ \\
Content Analysis & $25(23.6 \%)$ & $37(19.9 \%)$ & $62(21.2 \%)$ \\
Correlation & $14(13.2 \%)$ & $29(15.6 \%)$ & $43(14.7 \%)$ \\
ANOVA $/ t$ & $18(17.0 \%)$ & $21(11.3 \%)$ & $39(13.4 \%)$ \\
Regression & $10(9.4 \%)$ & $19(10.2 \%)$ & $29(9.9 \%)$ \\
Factor Analysis & $3(2.8 \%)$ & $13(7.0 \%)$ & $16(5.5 \%)$ \\
MANOVA & $3(2.8 \%)$ & $7(3.8 \%)$ & $10(3.4 \%)$ \\
Nonparametric & $4(3.8 \%)$ & $2(1.1 \%)$ & $6(2.1 \%)$ \\
\hline Totals & 106 & 186 & 292 \\
\hline
\end{tabular}


TPC consistently reported study effect sizes at a rate of $23.5 \%$ of all research articles published from 2011-2019: $F(1,152)=1.172 ; p=.281 ; \eta^{2}=.008$. Sample reliability and validity reports were also stable over time with reports of sample reliability at a steady rate of $39.2 \%-F(1,151)=1.961 ; p=.161 ; \eta^{2}=.012-$ and sample score validity at a consistent proportion of $36.6 \%$ from $2011-2019: F(1,151)=0.299 ; p=.585$; $\eta^{2}=.002$.

\section{Discussion}

TPC published an average of about 30 articles per year from 2011-2019, 57.7\% of which have been research articles. TPC mirrors the evolution of the counseling profession and through research and expository articles helps to address new professional issues, topics, and challenges that contribute to the advancement and growth of the counseling profession (TPC, 2020). The online publishing format makes this information freely available to professional counselors and the public; no print edition exists. This section discusses TPC author and article characteristics from the journal's inception in 2011 to 2019. Interestingly, only two variables displayed trends between the two time windows, demonstrating an admirable focus and stability of decision making among TPC editorial board members for a new counseling journal. That is, in just 9 years, TPC already appears to have attracted and published broadly consistent types of articles from authors with similar characteristics.

\section{Author Characteristics: Who Publishes in TPC?}

In the first 9 years of publication, only nine (3.4\%) TPC lead authors were domiciled outside the United States. Other counseling journals have also historically displayed international publishing rates of less than 5\%, including the Journal of Addictions $\mathcal{E}$ Offender Counseling (MacInerney et al., 2020), The Journal of Humanistic Counseling (Sylvester et al., in press), the Journal of College Counseling (Milowsky et al., in press), and the Journal of LGBT Issues in Counseling (Gayowsky et al., in press). Still, TPC has an opportunity to reach out to the international counseling community and invite more diverse expository and research contributions, thereby expanding into the international counseling domain. Perhaps a call for articles from international authors could form a special issue of TPC, contributing multicultural insights, evaluating cross-cultural perspectives, or helping to establish a focus for global connectivity. This is particularly important given NBCC's historic leadership in developing international counseling initiatives through its International Capacity Building department (NBCC, n.d.). International outreach is consistent with the mission of NBCC's international collaborations with organizations such as the African Union - Southern Africa Regional Office (AU-SARO) and around the world, and TPC is positioned to aid in that international scope of activities. As such, TPC could become a leader in international focus among counseling journals. Currently, two counseling journals do exceptional jobs attracting international scholars. The Journal of Employment Counseling (Siegler et al., in press) featured a 53\% internationally domiciled lead author proportion from 2010-2019, and Measurement and Evaluation in Counseling and Development published $28 \%$ of its articles from international lead authors over the same period (Saks et al., 2020).

A consistent majority of women composed the lead authors (64.2\%) and all authors (63.8\%) of published TPC articles from 2011-2019. A near 2-to-1 proportion is acknowledgement of the importance and majority standing of women counselor educator scholars in the United States (U.S. Census Bureau, 2016). A nearly two-thirds majority is consistent with many other counseling journals and indicates the rise to prominence of women scholars in counselor education (Johnson et al., 2021; MacInerney et al., 2020; Menzies et al., 2020; Milowski et al., in press; Saks et al., 2020; Sylvester et al., in press). 
At the same time the vast majority of lead authors (92.1\%) listed primary affiliations in university work settings. Although a non-university-affiliated participation rate of only $7.9 \%$ seems low overall, compared to other counseling journals, it actually is comparable with counseling journal leaders in this category like the Journal of Mental Health Counseling (Menzies et al., 2020) and Counseling Outcome Research and Evaluation (Johnson et al., 2021), and higher than most other counseling journals (MacInerney et al., 2020; Milowsky et al., in press; Saks et al., 2020; Sylvester et al., in press). This low level of participation from non-university-affiliated authors across counseling journals is curious given the powerful and necessary voices practitioners can lend to real-world counseling issues and interventions.

But there are certainly barriers and constraints on practitioner motivations to engage in scholarship. First, practitioners are rarely compensated for research, nor are employee evaluations substantively impacted by scholarly contributions. If more incentives like compensation and evaluation were tied to practitioner scholarship efforts, we could expect practitioners to have a larger presence in counseling scholarship and collaborations. Practitioners are also incredibly busy providing counseling services to clients and students, and research and program evaluation becomes a lower priority as time constraints tighten. In contrast, employee scholarship production often is rewarded by universities through salary increases, promotions, and tenure. Likewise, professors are expected to, and often rewarded for, scholarship, including collaboration with graduate students and colleagues in research projects. Graduate students often engage in research to enhance their levels of qualification for doctoral study and future opportunities to join the professoriate. It is also possible that authors who are practitionerstudents or scholar-practitioners simply default to a university affiliation for some reason-perhaps believing that university affiliations are more prestigious or may be given greater consideration in a positive disposition. In any case, journal editorial boards should encourage authors to list multiple affiliations to better gauge the prevalence of practitioners among contributing authors.

Counselor scholars should continue to search for opportunities to collaborate with practitioners, both to access rich sources of field-based data and to amplify the valuable voices of experienced clinicians. In just the first 9 years, TPC experienced increased author collaboration, from 2.43 authors per article in 2011-2014 up to 2.83 in 2015-2019. This trend toward more collaboration was seen in all other counseling journals, with many in the same vicinity of average author contributions as TPC, including AdultSpan (Rippeto et al., in press), the Journal of Employment Counseling (Siegler et al., in press), The Journal of Humanistic Counseling (Sylvester et al., in press), and others far higher, exceeding 3.10 authors per article, such as Measurement and Evaluation in Counseling and Development (Saks et al., 2020) and the Journal of College Counseling (Milowsky et al., in press).

Collaboration is essential to the future of counseling research as the profession and our domain of knowledge become larger and at the same time more specialized. This trend toward increased collaboration is very positive, perhaps reflecting a greater focus on mentorship and partnership. It will not be a surprise to note that simultaneous with this rise in collaboration was a proportional rise in publishing research articles. Research ventures are great opportunities for collaboration and building research partnerships with colleagues, graduate students, and practitioners. In particular, partnering with practitioners promotes meaningful field-based studies that enrich practice and document promising evidence-based interventions. Finally, as counselor educator-scholars, we must recommit to training practitioners who can collect, conduct, and collaborate in the publication of field-based research. Field-based studies are critical in counseling research because that is where the overwhelming majority of clients and students are counseled and treated. Conducting studies in 
research labs must generalize to implementation in the field; studies conducted in the field already have bridged that gap. Melding field-based innovations, counseling research, and practitioner voices will help the counseling profession evolve into a more evidence-based practice accountable to, and valued by, clients and other stakeholders.

\section{Article Characteristics: What Is Published in TPC?}

Over the first 9 years of TPC publications, articles were quite well balanced over a broad range of topical content (see Table 1). When combined, over one-third of the articles focused primarily on three topics: counselor education/training (16.4\%), multicultural issues (10.3\%), and school counseling $(10.3 \%)$. However, an appropriate and even spread of topic coverage was observed in the other areas, ranging from a low of $3.2 \%$ for identity development to $8.4 \%$ for health/wellness. Thus, the TPC editorial board is approving a broad swath of important and meaningful topical content for the readership of TPC. It will be important to maintain this balance in the decade ahead as TPC fulfills its goal as a journal of value to all professional counselors, regardless of work setting and counseling sub-discipline.

Research articles are critical components of professional journals. Research articles form the scientific foundation of a profession, and the more we know about the clients we serve, the better prepared we are to serve them. At 58.1\%, the proportion of research studies published in TPC is typical when compared with other counseling journals, most of which are in the range of $50 \%-68 \%$, including Counseling and Values: Spirituality, Ethics, and Religion in Counseling (Alder et al., in press); the Journal of LGBT Issues in Counseling (Gayowsky et al., in press); the Journal of Mental Health Counseling (Menzies et al., 2020); and Adultspan (Rippeto et al., in press). The proportion of research studies in TPC is albeit far lower than the more research-focused counseling journals like Measurement and Evaluation in Counseling and Development (80.7\%; Saks et al, 2020), Counseling Outcome Research and Evaluation (75.6\%; Johnson et al., 2021), the Journal of Employment Counseling (86.4\%; Siegler et al., in press), and Counselor Education $\mathcal{E}$ Supervision (90.4\%; Johnsen et al., in press) produced during the 2015-2019 time window. Still, TPC has made great inroads in the proportion of research articles published in its first decade, and it will be interesting to see where this trajectory will lead in the next decade.

The research designs used in TPC research studies are heavily weighted toward non-experimental designs, including descriptive or survey (42.4\%), qualitative (18.4\%), and correlational (18.4\%) designs (see Table 2). Descriptive/survey research simply seeks to describe the characteristics of participants and their interactions or performance on variables. True- and quasi-experimental designs appeared in only $4.4 \%$ of all TPC research articles, which is a low rate among the family of counseling journals. At the same time, the $12.3 \%$ proportion of intervention-focused articles is quite good when compared to other counselor journals (Menzies et al., 2020), as Ray et al. (2011) reported that only about 6\% of all research articles published in counseling journals focus on the efficacy of interventions. Intervention research studies tend to be among the most valuable studies published in the counseling profession because intervention studies inform the reader on what efficacy counselors can expect when implementing the intervention with clients or students. Thus, intervention studies help inform us about what we know that works in counseling. In summary, TPC should strive to increase the proportion of intervention articles, as well as true- and quasi-experimental designs, as these approaches help counselors understand what works in counseling related to the effectiveness of counseling practice. True- and quasi-experimental designs also enhance generalizability of sample results to the population, which gives counselors confidence that the more robust experimental results from these studies are more applicable to the broader populations of clients served by counselors. 
During its first 4 years of publication, TPC produced one of the highest proportions of qualitative tradition articles seen in the family of counseling journals (53.1\%), before declining significantly in 2015-2019 (30.7\%). Qualitative research has consistently accounted for 30-45\% of research publications in some counseling journals over the last 20 years (Alder et al., in press; Gayowsky et al., in press; MacInerney et al., 2020), but is much lower in other journals like Measurement and Evaluation in Counseling and Development (Saks et al, 2020) and Counseling Outcome Research and Evaluation (Johnson et al., 2021), which produce more quantitative, generalizable research. It will be interesting to see if the significant decline in qualitative articles continues over the next decade or stabilizes in the $30 \%$ range.

The significant increases in adults and counselors or other mental health service providers as participants in TPC research studies was interesting, as these two groups comprised more than twothirds (67.5\%) of participant types in the 2015-2019 time window (see Table 3). This was accompanied by a significant decline in the use of undergraduate and graduate students. The use of adult participants in counseling research is a welcome occurrence, as adults comprise the majority of clients seen in counseling agencies and private practice. And the focus on counseling practitioners is very appropriate for a journal whose mission it is to meet the professional needs of all counselors across diverse work settings and disciplines.

Although the median sample sizes did increase from 65 to 107 participants across the two time windows, that observation is expected given the increase in the proportion of quantitative compared to qualitative studies, as quantitative studies generally have higher sample sizes. Still, the overall consistency in the proportions of small, medium, large, and very large sample sizes is a sign of maturation in such a young journal.

Statistical procedures appearing in TPC articles were very well balanced across categories and across the two time windows. Not surprisingly, basic approaches like descriptive analyses and thematic coding comprised about half of all procedures. Descriptive statistics are the most basic type of statistics and are often used to convey results in surveys and nonexperimental studies. MANOVA, ANOVA, and $t$-tests combined for a very respectable total proportion of $16.8 \%$. TPC should strive to increase the prominence of these more sophisticated statistical tests. Among counseling journals, Counseling Outcome Research and Evaluation (Johnson et al., 2021) leads the way in use of these tests of difference statistics at more than $20 \%$, but TPC is not far behind.

Finally, TPC editors have a great deal of progress to make in improving the proportions of reporting standards. In the first 9 years of publication, TPC authors reported effect size estimates in only $23.5 \%$ of articles, sample reliability estimates in only $39.2 \%$, and sample validity estimates in $36.6 \%$ of research articles published. Reports of effect size, score reliability, and score validity are indications of study rigor and meaningfulness. For example, authors should always report on the reliability of scores of standardized dependent variables used in a study so readers know how much error variance in measurement occurred. Likewise, statistical significance is important in hypothesis testing, but effect sizes give readers an indication of how important and meaningful the results are in a grander context. These effect size and reliability reports are among the lowest rates of any counseling family journal (MacInerney et al., 2020; Milowsky et al., in press; Rippeto et al., in press; Saks et al., 2020; Siegler, in press; Sylvester et al., in press), so the TPC editorial board is encouraged to add these reporting standard aspects to review protocols and author requirements to insure inclusion in future articles. 


\section{Limitations and Conclusion}

This synthesis and analysis of the first 9 years of TPC publications has several limitations that should be explicated. First, no causal inference may be derived from these results. This means that one cannot determine the cause of any of the analyses indicated because the design of the study was not experimental; we were able to describe the variables, not determine what caused the results. This meta-study used descriptive and comparative statistical analyses to summarize proportions aggregated across time windows to discern trends occurring over time. However, no inference is offered as to why the proportions and trends were observed.

Second, despite the use of independent coders and robust, standardized coding procedures, the potential for coding errors always exists. Thus, some categorization or classification errors may have occurred. This is a particular risk within categories that are more subjective or when the descriptions provided by TPC authors lacked clarity or essential information. Third, we chose to segment the 9 years of TPC publications into two periods to bolster analytic power, although others might prefer smaller time windows when engaging in trend analysis.

Fourth, while this meta-study combined the quantitative and qualitative designs into a larger analysis and then took a closer look at just the quantitative designs, there is value in conducting a parallel analysis of the methodological rigor of both the quantitative and qualitative studies published in TPC to date. This will help address or at least acknowledge the possible limits of a positivist epistemological lens.

Finally, we were not able to code important author demographics such as race, ethnicity, age, or other attributes. We instead focused on readily available information like lead author domicile and author institutional affiliations and gender. For future analyses, it would be helpful if authors would self-identify a wider variety of demographic descriptors (e.g., self-identified gender, race, nationality) or at least designate all university and non-university affiliations. This would help clarify the degree to which practitioners and authors of diverse backgrounds are represented within author configurations.

In summary, TPC has made great progress across many dimensions over its first 9 years of publication. Also, it appears that TPC is well on its way to fulfilling its mission to provide valuable research and practice information to all professional counselors regardless of work setting or sub-discipline. We look forward to seeing the continued progress of this new counseling journal in the decades ahead and hope this meta-study is of use to the TPC editorial board, authors, and readership as a description of who and what is published in TPC.

\section{Conflict of Interest and Funding Disclosure}

The authors reported no conflict of interest or funding contributions for the development of this manuscript. 


\section{References}

Alder, C., Yu, C., \& Erford, B. T. (in press). A meta-study of the Counseling and Values: Spirituality, Ethics, and Religion in Counseling (CVJ): An analysis of publication characteristics, 2000-2019. Counseling and Values: Spirituality, Ethics, and Religion in Counseling.

Erford, B. T., Miller, E. M., Duncan, K., \& Erford, B. M. (2010). Submission patterns: Measurement and Evaluation in Counseling and Development author and article characteristics from 1990 to 2009. Measurement and Evaluation in Counseling and Development, 42(4), 296-307. https://doi.org/10.1177/0748175609354619

Gayowsky, J., Choi, J., \& Erford, B. T. (in press). Meta-study of Journal of LGBT Issues in Counseling publication patterns from 2006-2019. Journal of LGBT Issues in Counseling.

Johnsen, S., Watson, K., Erford, B. T., Crockett, S., \& Byrd, R. (in press). Counselor Education and Supervision (CES): An analysis of publication characteristics from 2000-2019. Counselor Education and Supervision.

Johnson, A., West, C., \& Erford, B. T. (2021). A meta-study of Counseling Outcome Research and Evaluation (CORE): An analysis of publication characteristics from 2010-2019. Counseling Outcome Research and Evaluation.

MacInerney, E. K., Johnsen, S. T., Cobb, N., Smith, H., \& Erford, B. T. (2020). Journal of Addictions E Offender Counseling publication patterns from 1998 to 2017: Author and article characteristics. Journal of Addictions $\mathcal{E}$ Offender Counseling, 41(2), 82-96. https://doi.org/10.1002/jaoc.12082

Menzies, A., Kepley, L., Crockett, S. A., Erford, B. T., Byrd, R., \& Aier, B. (2020). A meta-study of the Journal of Mental Health Counseling: An analysis of publication characteristics 2000-2019. Journal of Mental Health Counseling, 42(3), 206-219. https://doi.org/10.17744/mehc.42.3.02

Milowsky, A. I., Haffner, A. M., Bardhoshi, G., Erford, B. T., Byrd, R., \& Crockett, S. A. (in press). A meta-study of Journal of College Counseling (JCC): An analysis of publication characteristics from 2000-2019. Journal of College Counseling.

National Board for Certified Counselors. (n.d.). NBCC Foundation: International capacity building. https://www. nbccf.org/programs/international

The Professional Counselor. (n.d.). The Professional Counselor. https://tpcjournal.nbcc.org

Ray, D. C., Hull, D. M., Thacker, A. J., Pace, L. S., Swan, K. L., Carlson, S. E., \& Sullivan, J. M. (2011). Research in counseling: A 10-year review to inform practice. Journal of Counseling E Development, 89(3), 349-359. https://doi.org/10.1002/j.1556-6678.2011.tb00099.x

Rippeto, M., Stewart, E., \& Erford, B. T. (in press). A meta-study of Adultspan publication characteristics from 1999-2019. Adultspan.

Saks, J., Fuller, A., Erford, B. T., \& Bardhoshi, G. (2020). Meta-study of Measurement and Evaluation in Counseling and Development (MECD) publication patterns from 2000-2019. Measurement and Evaluation in Counseling and Development, 53(4), 279-288. https://doi.org/10.1080/07481756.2020.1735222

Siegler, E., Stafford, C., Crockett, S., Zhou, Y., \& Erford, B. T. (in press). A meta-study of Journal of Employment Counseling (JEC): An analysis of publication patterns from 2010-2019. Journal of Employment Counseling.

Sylvester, A.-M., Wary, S., Erford, B. T., \& MacInerney, E. (in press). A meta-study of The Journal of Humanistic Counseling (JHC): An analysis of publication characteristics, 2000-2019. Journal of Humanistic Counseling.

U.S. Census Bureau. (2016). ACS public use microdata sample. [Data file]. https://www.census.gov/programssurveys/acs/data/pums.html 